ESAIM: PROCEEDINGS AND SURVEYS, December 2016, Vol. 55, p. 131-147

Emmanuel FRÉNOD, Emmanuel MAITRE, Antoine ROUSSEAU, Stéphanie SALMON and Marcela SZOPOS Editors

\title{
NON CLASSICAL SOLUTION OF A CONSERVATION LAW ARISING IN VEHICULAR TRAFFIC MODELLING*
}

\author{
Mathieu Fabre ${ }^{1}$, Sylvain Faure ${ }^{2}$, Mathieu Laurière ${ }^{3}$, Bertrand \\ MAURY $^{4}$ AND CHARlOtTE PERRIN ${ }^{5}$
}

\begin{abstract}
We are interested in this paper in the modelling and numerical simulation of some phenomena that are observed in the context of car dynamics, in particular the appearance of persistent jams upstream critical points with no real cause of flux limitation. We shall consider the case of a stable jam on a freeway upstream an accident that took place on the opposite lane. This situation is not properly handled by most models, either micro-or macroscopic ones, since it corresponds to a phenomenon that does not have a counterpart in gas dynamics, for which only entropy solution are usually considered as physically feasible. The approach we propose consists in accounting for the very behaviour of agents in the neighbourhood of the discontinuity, and makes it possible to numerically recover in a robust way steady traffic jams.
\end{abstract}

Résumé. Nous nous intéressons dans cet article à la modélisation et à la simulation numérique de phénomènes particuliers observés dans le contexte du trafic routier, plus particulièrement le phénomène d'apparition et de persistance de bouchons en amont de points critiques sans cause objective de ralentissement. Nous considérerons notamment le cas d'un bouchon persistant sur une autoroute en amont d'un accident qui s'est produit sur la voie d'en face. Cette situation n'est pas reproduite par la plupart des modèles, qu'ils soient microscopiques ou macroscopiques, car elle correspond à un phénomène qui n'a pas d'équivalent en dynamique des gaz, pour lesquels seules les solutions dites entropiques sont en général considérées comme correspondant à un comportement observable dans la réalité. L'approche que nous proposons, aux niveaux microscopique et macroscopique, consiste à prendre en compte le comportement particulier des agents humains au voisinage de la discontinuité, et permet de retrouver numériquement de façon robuste des bouchons stables.

\section{INTRODUCTION}

Modelling traffic has become in the recent years a major challenge for the applied mathematics community. Among all the issues raised by vehicular traffic behaviours we are particularly interested in the modelling of congestion phenomenon, how traffic jams appear, persist and disappear. There are mainly three approaches to model traffic motion, each corresponding to a specific scale: microscopic scale (each entity is individually followed), macroscopic scale (the collection of entities

\footnotetext{
* Acknowledgements : The authors would like to thank F. Lagoutière for his fruitful suggestions. This project is supported by ANR Project Isotace (ANR-12-MONU-0013).

${ }^{1}$ EPFL SB MATHICSE MNS (Bât. MA), station 8, CH 1015 Lausanne (Switzerland), mathieu.fabre@epfl.ch

${ }^{2}$ Laboratoire de Mathématiques d'Orsay, CNRS, Univ. Paris-Sud, Université Paris-Saclay, 91405 Orsay, France.

${ }^{3}$ NYU-ECNU Institute of Mathematical Sciences at NYU Shanghai (China), mathieu.lauriere@nyu.edu

${ }^{4}$ LMO, Univ. Paris-Sud, Université Paris-Saclay, 91405 Orsay, France \& DMA, École Normale Supérieure, Paris

${ }^{5}$ Institut für Mathematik, RWTH Aachen University, perrin@instmath.rwth-aachen.de
}

(c) EDP Sciences, SMAI 2017 
is described by a local density), and mesoscopic scale (kinetic description). In this paper we will focus on the first two descriptions, the interested reader is referred to 4 for a review of the kinetic models.

The most direct way to describe the motion of a collection of vehicles is to represent each of them individually and to follow the trajectory of each agent, taking into account all the interactions with the surrounding agents. In one-dimensional situations (a one-lane road or a corridor), a simple description of the dynamics is provided by Follow-The-Leader models used for instance in [5] - 12. In this context congestion is handled by introducing short-range correction terms which mimic some repulsion when an agent gets closer to the agent next to him. This is the approach adopted in Follow-The-Leader models, where the velocity is supposed to decrease with the inter-agent distance, and is taken to be zero at a minimal distance corresponding to the size of the agents, which is not negligible in the discrete description. Note that such models are not based on classical mechanics principles: interaction terms are not symmetric, since each agent is influenced by the one that lies in front of him, with no reciprocity.

Another mathematical description widely used consists in considering the assembly of vehicles as a continuous medium, and working with averaged variables: the average velocity of agents in an elementary volume (or length in the one-dimensional setting), and the density, which corresponds to the number of agents per unit volume. We then make an analogy with fluids by writing a balance equation expressing at the macroscopic level the conservation of the number of agents. If one wants to simulate the behaviour of a large number of agents, the macroscopic description may be attractive compared to the microscopic description for the computational cost and for the relative ease with which one can calibrate the model due to the low number of parameters. As we shall see, although such models are based on principles that are similar to the Follow-TheLeader approach, the "directionality of influence" that we previously mentioned will disappear in the expression of interaction terms. This very distinction between the two approaches will play a crucial role in the present paper.

Intuitively, discrete models like the Follow-The-Leader models should converge towards continuous conservation laws. This issue has been the object of few and relatively recent mathematical works. In [3], the authors prove a mathematical link between the discrete solutions of a second order (in time) Follow-The-Leader model for car traffic, and the semi-discretization in time of a macroscopic solution to a system of conservation laws called Aw-Rascle model. The formal idea consists in letting the number of vehicles go to infinity while imposing a particular scaling in time and space which ensures that the density and the velocity remain fixed.

Concerning first order Follow-The-Leader models, which neglect the effects of inertia in the motion, this question has been treated by Colombo and Rossi in $[8$ and by Di Francesco and Rosini in [11. In the latter, the authors prove that the discrete solutions satisfy a discrete inequality of Oleinik's type which yields strong controls of the semi-discrete density, and the strong convergence towards the unique entropy solution of the limit conservation law.

This limit passage between the microscopic and the macroscopic scales is also related to homogenization techniques. Such techniques have been interestingly used for instance by Forcadel, Imbert, Monneau 9 to prove the convergence of the primitive of the semi-discrete density towards a viscosity solution of a macroscopic Hamilton-Jacobi equation (which can be seen as a primitive of the LWR model). In a recent paper, Forcadel and Salazar [10 study this limit passage for a FTL model with a local perturbation depending on the position which leads at the macroscopic limit to a local flux limiting condition.

In this paper, we are interested in particular phenomena that can be observed and experimentally reproduced for cars, the typical situation we have in mind is the following: an accident takes place on a freeway, and instantaneously stops the traffic on both lanes. Traffic is frozen upstream the accident, and a jam is also created on the opposite lane due to drivers' curiosity. Although there is no physical obstacle on this lane, it can be commonly observed that this jam persists until every trace of the accident has been removed. As it will be detailed, macroscopic and microscopic 
standard models fail to reproduce this persistence of a jam. In [17, Polsen and Sokolov develop a particle filtering and learning algorithm to estimate and update the parameters of their macroscopic model. Applying it to the previously described situation, they are then able to identify a drop in the road capacity (i.e. the critical/maximal flow) and reproduce the formation of a jam on the opposite lane. Contrary to them, we want to keep the parameters of the road fixed. We propose here to model this type of phenomenon at the microscopic level by locally perturbing the classical Follow-The-Leader model. We shall consider that the behavior of the agents is modified in a small buffer zone, that corresponds to the zone in which the accident in the opposite lane can be seen. We can interpret the modification as follows: when entering in direct visual contact with the accident, people cannot help looking at it, and they no longer look carefully at the vehicle upfront, that may already be accelerating. Yet, because of the presence of police forces or because of moral considerations, they do not completely stop. We shall consider that they keep the velocity they had when entering in the buffer zone. This perturbation is different from the one introduced by Forcadel and Salazar in [10]. In their paper, the velocity is modulated by an additional function of the position, while here, the dynamics itself is modified in the buffer zone since the acceleration is imposed to be zero.

By letting the number of agents increase, the numerical solution is expected to converge to a solution of a macroscopic conservation law. The situation we will obtain, as expected, corresponds to what is observed in practice: a congested zone upstream the "accident", at low velocity, and a dilute zone downstream, at high velocity. Since characteristics go leftward on the left hand side of the discontinuity, and rightward on the right hand side, it corresponds to a non-classical solution, i.e. a non entropy solution, whereas the entropy solutions are usually considered as the "physical solutions" (see Ansorge 2]) and the most common numerical schemes are built to select this type of solutions. Non-classical solutions have been already considered for traffic (or pedestrian) flow modelling, but in other contexts: for instance when a additional flux constraint is imposed (see [6], [1]), or to describe panic phenomena in a crowd (see [7]).

Directly considering the macroscopic model, we will show that a local modification of a standard numerical scheme (originally consistent with the entropy conditions), is sufficient to capture stable traffic jams.

\section{MiCROSCOPIC MODELS FOR TRAFFIC JAMS}

We consider the one-dimensional domain $\mathbb{T}=[0,2 \pi]$ supplemented by periodic boundary conditions. By doing so we aim at modelling the dynamics of vehicles on a circular road. We will focus in this section on a first order Follow-The-Leader model (denoted FTL) where the vehicles instantaneously reach the desired velocity, which is a given function of the distance with the upfront vehicle. Starting with an initial congested distribution of vehicles on the circular road, we will show that the FTL model predicts a relaxation towards the uniform distribution with constant velocity for all the vehicles and constant distances between them. We will then try to perturb this dynamics by introducing a buffer zone which corresponds to the zone where the drivers look at the opposite lane and forget to follow the previous car. Finally, by letting numerically the number of cars increase, we want to approximate the continuous density of vehicles which is a solution to an appropriate conservation law.

\subsection{The discrete Follow-the-Leader model}

The Follow-The-Leader (FTL) model has been first introduced to model car traffic. It expresses the fact that each driver follows the car in front of him by adjusting his speed according to the distance between them. Let us consider $N$ cars, whose positions and speeds are denoted respectively by $x_{i}$ and $v_{i}, i=1, \ldots, N$, where the $(i+1)$ st car follows the $i$ th car. Then the FTL model consists of $N$ differential equations: for $i=1, \ldots, N$ :

$$
\frac{\mathrm{d} x_{i+1}}{\mathrm{~d} t}(t)=v_{i+1}(t)=\varphi\left(\delta_{i}(t)\right),
$$


where $\delta_{i}=x_{i}-x_{i+1}$ is the distance between the $i$-th car and the following one. By periodicity we define $\delta_{N}=x_{N}-x_{1}$. The function $\varphi$ is assumed to be concave, increasing and with nonnegative values. Here, we will consider the particular function

$$
\varphi(\delta)=v_{\max }\left(1-\frac{\delta^{*}}{\delta}\right) \mathbf{1}_{\left\{\delta>\delta^{*}\right\}},
$$

where $\delta^{*}>0$ represents the size of a car (all the cars are assumed to have the same size), and $v_{\max }$, the maximal velocity, is the velocity the cars would have if there were nobody in front of them. We have represented this function on Figure 1 . The interested reader is referred to 12

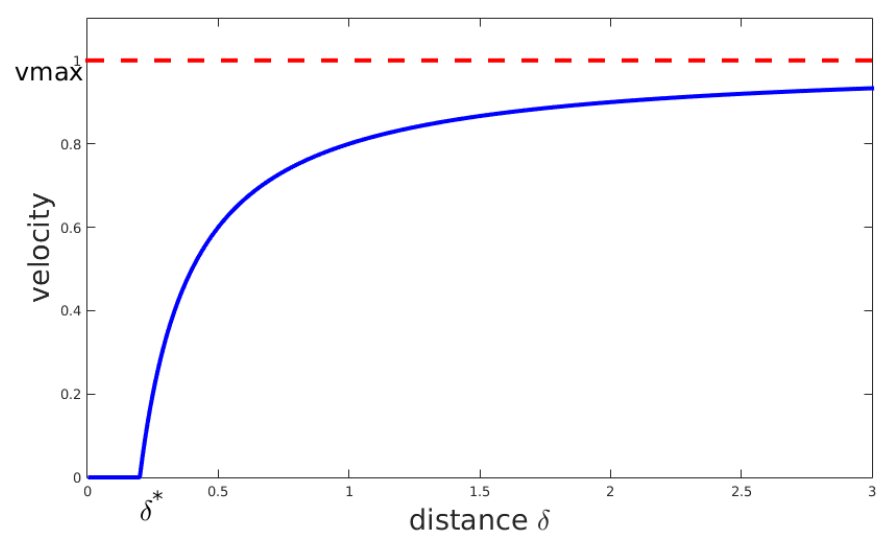

Figure 1. Velocity of the FTL model. Parameters: $v_{\max }=1$ and $\delta^{*}=0.1$.

concerning general FTL models.

The equations (1) can be rewritten as: for $i=1, \ldots, N$ :

$$
\frac{\mathrm{d} \delta_{i}}{\mathrm{~d} t}(t)=\varphi\left(\delta_{i-1}(t)\right)-\varphi\left(\delta_{i}(t)\right) .
$$

Equilibrium of the basic system. Associated to this model, there exists a unique periodic solution which corresponds to the uniform distribution of the distances. All the distances and the velocities are then respectively equal to

$$
\delta_{\mathrm{eq}}=\frac{2 \pi}{N}, \quad v_{\mathrm{eq}}=v_{\max }\left(1-\frac{\delta^{*}}{\delta_{\mathrm{eq}}}\right) .
$$

In the previous equations, we implicitly assume that the number of vehicles on the road is not too large, namely

$$
N<\frac{2 \pi}{\delta^{*}}
$$

Introducing the discrete entropy

$$
S(\Delta)=\sum_{i} \delta_{i} \log \delta_{i}, \quad \text { for } \quad \Delta=\left(\delta_{i}\right)_{i=1, \ldots, N}
$$

one can recover that the uniform distribution is a global attractor of the system. Indeed, one can show that the discrete entropy decreases in time and is minimal for the uniform distribution. Let us recall the main steps allowing to prove this fact. By replacing the distance $\delta_{i}$ by $\frac{\delta_{i}}{2 \pi}$ we can 
assume that $\sum \delta_{i}=1$. Differentiating the entropy $S$ with respect to time and using the periodicity, we have

$$
\begin{aligned}
\frac{\mathrm{d}}{\mathrm{d} t} S(\Delta) & =\sum_{i}\left(\log \delta_{i}+1\right) \frac{\mathrm{d}}{\mathrm{d} t} \delta_{i} \\
& =\sum_{i}\left(\log \delta_{i}+1\right)\left(\varphi\left(\delta_{i-1}\right)-\varphi\left(\delta_{i}\right)\right) \\
& =\sum_{i} \log \delta_{i}\left(\varphi\left(\delta_{i-1}\right)-\varphi\left(\delta_{i}\right)\right) .
\end{aligned}
$$

If the distribution is uniform, then we get 0 . Otherwise, we can split the sum into two parts depending on the sign of $\delta_{i-1}-\delta_{i}$ :

$$
I_{1}=\left\{(i-1, i), \delta_{i-1}<\delta_{i}\right\}, \quad I_{2}=\left\{(i-1, i), \delta_{i-1} \geq \delta_{i}\right\} .
$$

The strict concavity of the function $\varphi$ leads to

$$
\frac{\mathrm{d}}{\mathrm{d} t} S(\Delta)<\varphi^{\prime}(1) \sum_{I_{1}} \log \left(\delta_{i}\right)\left(\delta_{i-1}-\delta_{i}\right)+\varphi^{\prime}\left(\delta^{*}\right) \sum_{I_{2}} \log \left(\delta_{i}\right)\left(\delta_{i-1}-\delta_{i}\right) .
$$

Rearranging the sums we obtain

$$
\begin{aligned}
\frac{\mathrm{d}}{\mathrm{d} t} S(\Delta) & <\varphi^{\prime}(1)\left(\sum_{i} \log \left(\delta_{i}\right)\left(\delta_{i-1}-\delta_{i}\right)\right)+\left(\varphi^{\prime}\left(\delta^{*}\right)-\varphi^{\prime}(1)\right) \sum_{I_{2}} \log \left(\delta_{i}\right)\left(\delta_{i-1}-\delta_{i}\right) \\
& <\varphi^{\prime}(1)\left(\sum_{i} \log \left(\delta_{i}\right)\left(\delta_{i-1}-\delta_{i}\right)\right) \\
& <\varphi^{\prime}(1) \sum_{i} \log \left(\frac{\delta_{i+1}}{\delta_{i}}\right) \delta_{i} .
\end{aligned}
$$

Using the fact that $\sum_{i} \delta_{i}=1$ and the concavity of the logarithm we finally obtain

$$
\frac{\mathrm{d}}{\mathrm{d} t} S(\Delta)<\varphi^{\prime}(1) \log \left(\sum_{i} \frac{\delta_{i+1}}{\delta_{i}} \delta_{i}\right)=\varphi^{\prime}(1) \log \left(\sum_{i} \delta_{i+1}\right)=0 .
$$

The entropy is therefore a global and strict Lyapunov functional for the considered equilibrium point, which asserts global asymptotic stability.

Behaviour in presence of high density. Starting from an initial distribution where cars are concentrated in a small region, the system evolves towards the stable solution where the distances are uniform. In other words, a relaxation leads to the dissolution of the jam, as illustrated by Figure 2 .

We have represented the trajectories of some cars, as well as the distances between them and the cars in front of them. We observe that after some time, the trajectories coincide with lines with a common slope which is the inverse of the uniform velocity $v_{\text {eq. }}$. After some oscillations, the distances seem to converge towards the uniform distance $\delta_{\text {eq }}$.

\subsection{Inclusion of a buffer zone}

In order to model the persistence of some traffic jams, we propose to include in the domain a buffer zone where the velocities are "frozen". In other words, drivers go through this zone with a constant velocity, equal to the velocity they had when they entered this buffer zone. This encodes the following behavior: the driver looks at the accident on the opposite lane, while maintaining his place. Let us denote $Z_{F T L}$ the normal zone where the drivers behave according to the FTL model, and $Z_{f}=\mathbb{T} \backslash Z_{F T L}$ the buffer zone. 

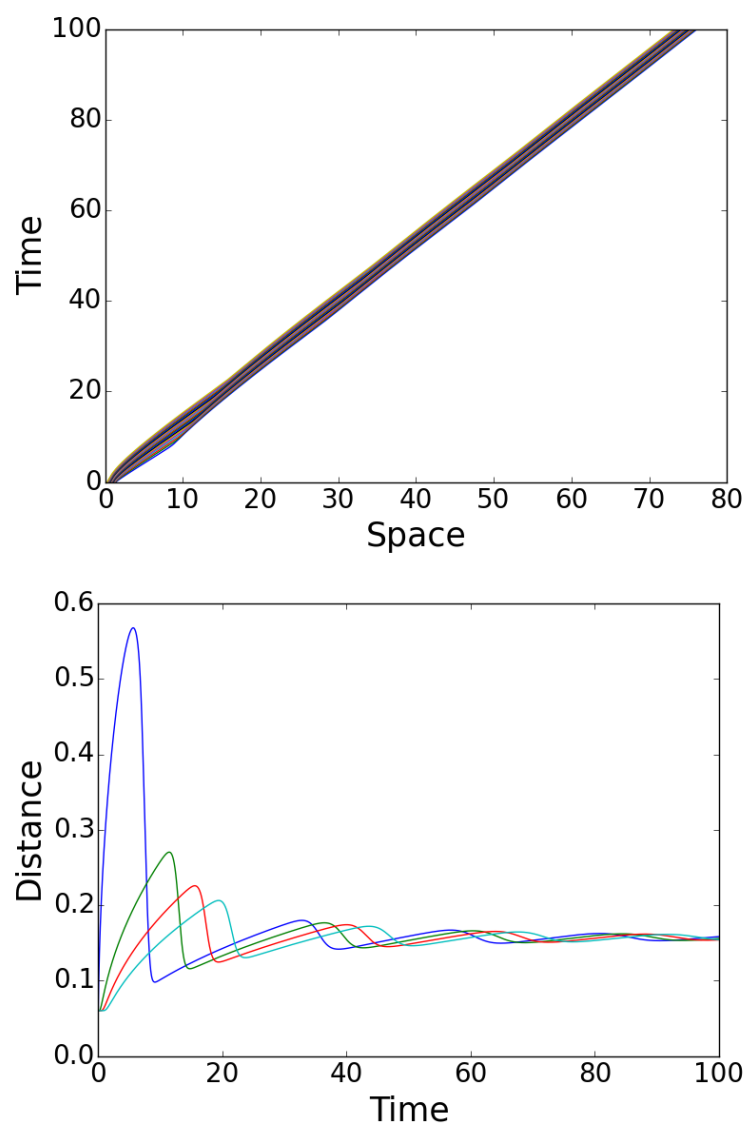

Figure 2. Relaxation of the FTL model ( $N=40$ vehicles) which can be seen on the trajectories (on the left: for one out of every two vehicles), or on the evolution of the distances (on the right: for four cars: $i=1,10,20$ and 40). Parameters: $v_{\max }=1$ and $\delta^{*}=0.04$.

The model writes: for every $i=1, \ldots, N$

$$
\begin{cases}\frac{\mathrm{d} x_{i+1}}{\mathrm{~d} t}(t)=\varphi\left(\delta_{i}(t)\right) & \text { if } \quad x_{i+1} \in Z_{F T L} \\ \frac{\mathrm{d}^{2} x_{i+1}}{\mathrm{~d} t^{2}}(t)=0 & \text { if } \quad x_{i+1} \in Z_{f}\end{cases}
$$

Size of the buffer zone. In order to see an effect of this zone on the dynamics, we need to ensure that there is always at least one car whose velocity is frozen. The buffer zone then needs to be large enough compared with the size $\delta^{*}$ of the cars, the expected velocity in traffic jam $v_{i}$, and the time step $\Delta t$.

In our simulations we have fixed the length of the frozen zone $L_{f}$ as

$$
L_{f}=3 \delta^{*},
$$

and we have taken the time step $\Delta t$ such that it is much smaller than the critical time step $\Delta t_{c}$ :

$$
\Delta t \ll \Delta t_{c}=\frac{L_{f}}{v_{i}} .
$$


We can thereby ensure that the cars spend several time steps in the buffer zone, which can modify the FTL dynamics.

Behaviour in presence of high density. Starting from an initial situation where the buffer zone is congested, we expect the system to remain congested. Intuitively, this is for the following reason: when a driver enters the buffer zone, he is stuck in the jam so his speed is very low. Then he slowly passes through the buffer zone, and even when the vehicle in front of him exits from the buffer zone and accelerates due to the dynamics imposed by the FTL model, he will keep the same speed. This maintains the jam behind him. Thus, locally freezing the car response can be expected to maintain the presence of a jam.

We observe on the simulations that we do not have any more the relaxation process as in the normal FTL model (see Figure 2), and it seems that the traffic jam is persistent. On Figure 3 are represented the trajectories of the cars. After some time, we observe periodicity of the movement which corresponds to a stable traffic jam.

We have also represented on Figure 3 the velocities of four following cars from the time they enter into the traffic jam until they leave the buffer zone. We observe first an alignment of the velocities with the velocity imposed initially on the traffic jam. In this regime, the evolution of the velocities is continuous due to the FTL dynamics. Then, the driver enters into the buffer zone. When he finally leaves the buffer zone, he is subject to a speed jump which corresponds to the transition between the buffer zone and the FTL dynamics.
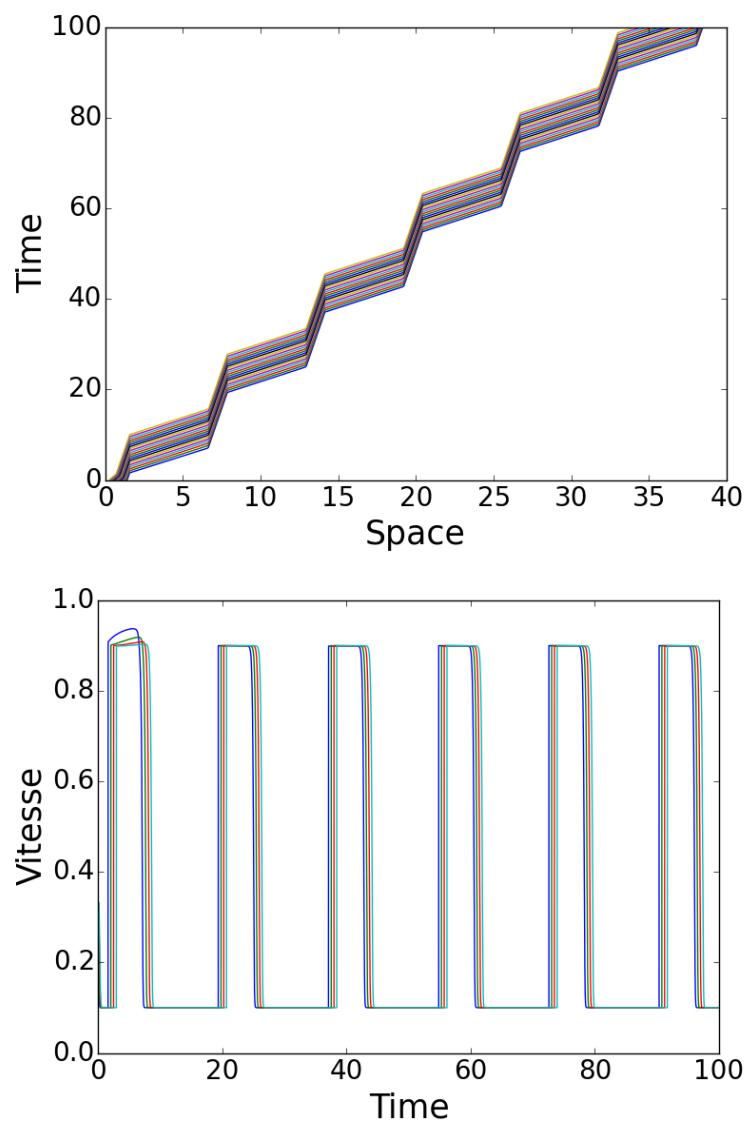

Figure 3. Trajectories (on the left: for one out of every two vehicles), and evolution of the velocities of four following vehicles (on the right). Parameters: $N=40, v_{\max }=1$ and $\delta^{*}=0.04$. 


\subsection{Macroscopic limit passage}

Basing our numerical investigations on the recent paper of Colombo and Rossi [8], we introduce the micro-macro operator for a fixed integer $N$ :

$$
C_{N}: X=\left(\begin{array}{c}
x_{1} \\
\vdots \\
x_{N}
\end{array}\right) \mapsto \rho_{N}=\sum_{i} \frac{\delta_{N}^{*}}{x_{i}-x_{i+1}} \mathbf{1}_{\left[x_{i+1}, x_{i}\right]},
$$

where $\delta_{N}^{*}$ is the length of a car (and so it corresponds to the minimal distance between two cars). This operator $C_{N}$ associates to the discrete distribution $X$ of the vehicles a piecewise constant density $\rho_{N}$. We expect $\rho_{N}$ to converge almost everywhere when $N \rightarrow+\infty$ to the solution of a macroscopic conservation law.

For our simulations we set

$$
\delta_{N}^{*}=\frac{1}{N}
$$

so that $N \delta_{N}^{*}$ is constant. The size $L_{f}$ of the buffer zone is, as previously, related to the size of the vehicles by

$$
L_{f}=3 \delta_{N}^{*} .
$$

Note that this quantity converges to 0 as the number of cars $N$ goes to infinity. Formally, we expect then that the microscopic model coupling FTL dynamics and the buffer zone converges towards the same macroscopic model as the single FTL model. This conservation law is

$$
\partial_{t} \rho+\partial_{x}(\rho v(\rho))=0 .
$$

In order to link this macroscopic velocity $v$ with the velocity $\varphi$ of the discrete FTL model we express the compatibility between the two descriptions on the discrete density $\rho_{N}$ (see for instance 8 eq. (2.4)). Let $x \in[0,2 \pi]$; there exists $i \in\{1, \ldots, N\}$ such that $\rho_{N}(x)=\delta^{*} / \delta_{i}$, replacing then $\delta_{i}$ in the expression of the velocity of the FTL model we get

$$
\varphi\left(\delta_{i}\right)=\varphi\left(\frac{\delta^{*}}{\rho_{N}(x)}\right)=v_{\max }\left(1-\frac{\delta^{*}}{\delta^{*} / \rho_{N}(x)}\right)=v_{\max }\left(1-\rho_{N}(x)\right) .
$$

The velocity involved in the limit conservation law is therefore $v(\rho)=v_{\max }(1-\rho)$.

We have represented in Figure 4 the discrete density $\rho_{N}$ computed from the model coupling the FTL dynamics and the buffer zone. The profile of this discrete density seems to be stable. It consists of a high density zone before and in the buffer zone corresponding to the traffic jam and of a low density zone outside the traffic jam.

We want to recover in the next section this type of solution directly on the limit conservation law.

\section{Conservation laWs FOR CONGESTiOn}

In the previous section we have let numerically the number of vehicles increase and thus constructed an approximate solution of a macroscopic model for a traffic jam. Historically, macroscopic systems based on the principles of the continuum mechanics have been first introduced for traffic flow modelling. The most famous model has been derived in 1955 by Lighthill and Whitham [16] and completed one year later by Richards [18 who added the notion of shock wave in the model. This system, still widely used, is commonly called LWR model. It expresses the physical principle of mass conservation for the assembly of vehicles

$$
\partial_{t} \rho+\partial_{x}(\rho u)=0
$$

on the closed and bounded interval $\Omega$, where $\rho$ denotes the density of cars, $u$ the mean velocity of the agents in an elementary volume. Both are considered as functions of time and space. We 


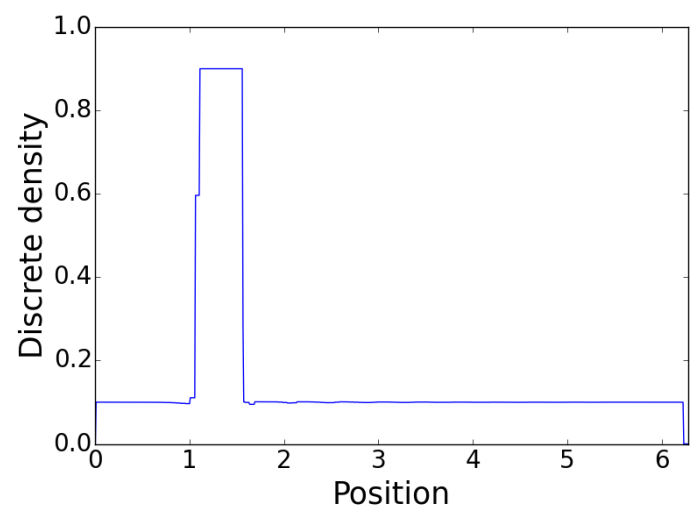

Figure 4. Discrete density $\rho_{N}$ for $N=200$ cars, stationary state. Parameters: $v_{\max }=1$ and $\delta^{*}=1 / N=0.005$, end of buffer zone at $x=1.5$.

prescribe the velocity $u$ as a function of the density: it will be the product of a desired velocity $U$ and a term, denoted $\beta(\rho)$, representing the effects of saturation

$$
\partial_{t} \rho(t, x)+\partial_{x}(\rho(t, x) \beta(\rho(t, x)) U)=0, \quad(t, x) \in[0, T] \times \Omega .
$$

The function $\beta$ is then assumed to be strictly decreasing on $[0,1]$. In the literature several expressions have been proposed for the function $\beta$. Among them one can cite those of

- Greenshields: $\beta(\rho)=1-\rho$,

- Pipes-Munjal: $\beta(\rho)=1-\rho^{\alpha}, \alpha>0$,

- Underwood: $\beta(\rho)=e^{-\rho}$,

- Greenberg (modified): $\beta(\rho)=\left[\log \left(\frac{1}{\alpha}\right)\right]^{-1} \log \left(\frac{1}{\rho+\alpha}\right), \alpha>0$.

The functions $\beta$ are chosen to fit with the so-called fundamental diagram which represents the experimental flux of cars as a function of their density as explained in [15] or [4]. Our previous study on the microscopic system (see $\S 1.3$ leads us to consider a function of Greenshields type,

$$
\beta(\rho)=1-\rho .
$$

In the rest of this section we will first describe a new numerical scheme for dealing with congestion effects in the one-dimensional periodic setting. We prove that the numerical scheme is appropriate for entropy solutions and therefore does not capture the stable congested numerical solutions which has been exhibited in the previous section. We then propose a local modification of the scheme which reproduces at the macroscopic level the ideas of the microscopic buffer zone.

\subsection{Upwind/downwind numerical scheme}

We consider the domain $\Omega=\mathbb{T}$, which denotes the one-dimensional interval $[0,2 \pi]$ with periodic boundary conditions as it has been done in the microscopic setting (see Section 1). It means that there is one entrance at $x=0$ and one exit at $x=2 \pi$, with an inflow at $x=0$ equal to the outflow at $x=2 \pi$. In this situation the desired velocity can be considered as a positive constant, which we take to be $U=1$ for simplicity.

Our numerical scheme is a finite volume scheme which aims at reproducing the physical transport of the information. The information of the transport of the mass is propagating upwind (since the mass moves forward), whereas the information of congestion can be seen as an information propagating downwind. Our scheme is based on a separate treatment of these two features.

We discretize the interval $[0,2 \pi]$ with a uniform grid of step size $\Delta x$, and discretize the time scale $[0, T]$ uniformly with a step size $\Delta t$, such that $1 / \Delta x$ and $T / \Delta t$ are two integers, denoted respectively by $N_{x}$ and $N_{t}$. The points in space and time are indexed in $\left\{1, \ldots, N_{x}\right\}$ and $\left\{0, \ldots, N_{t}\right\}$ 
respectively. Let $\rho_{i}^{n} \in \mathbb{R}$ be the approximation of $\rho$ on $[(i-1) \Delta x, i \Delta x]$ at time $n \Delta t$. Our explicit scheme then writes: for each $i \in\left\{2, \ldots, N_{x}-1\right\}$,

$$
\frac{\rho_{i}^{n+1}-\rho_{i}^{n}}{\Delta t}+\frac{1}{\Delta x}\left[\rho_{i}^{n} \beta\left(\rho_{i+1}^{n}\right)-\rho_{i-1}^{n} \beta\left(\rho_{i}^{n}\right)\right]=0,
$$

with the periodic boundary condition

$$
\rho_{1}^{n} \beta\left(\rho_{1}^{n}\right)=\rho_{N_{x}}^{n} \beta\left(\rho_{N_{x}}^{n}\right) .
$$

Properties of the numerical scheme. Our numerical scheme is under conservative form as we can see by writing

$$
\begin{gathered}
\rho_{i}^{n+1}=\rho_{i}^{n}-\frac{\Delta t}{\Delta x}\left(g_{i+1 / 2}^{n}-g_{i-1 / 2}^{n}\right), \\
\text { with } \quad g_{i+1 / 2}^{n}=g\left(\rho_{i}^{n}, \rho_{i+1}^{n}\right)=\rho_{i}^{n} \beta\left(\rho_{i+1}^{n}\right) .
\end{gathered}
$$

We directly check that the scheme is consistent, i.e. $g(\rho, \rho)=\rho \beta(\rho)$. Moreover, we argue that it is monotone under the CFL condition

$$
\frac{\Delta t}{\Delta x}\left(1+\max _{w \in[0,1]}\left|\beta^{\prime}(w)\right|\right) \leq 1
$$

(for $\beta(\rho)=1-\rho$, the condition is simply: $\Delta t \leq \Delta x / 2$ ). Indeed, we recall that (see for instance the reference book written by Godlewski and Raviart [13) a numerical scheme

$$
\rho_{i}^{n+1}=H\left(\rho_{i-1}^{n}, \rho_{i}^{n}, \rho_{i+1}^{n}\right),
$$

is said to be monotone if the function $H: \mathbb{R}^{3} \rightarrow \mathbb{R}$ is an increasing function in each argument. In our case,

$$
H\left(v_{1}, v_{2}, v_{3}\right)=v_{2}-\frac{\Delta t}{\Delta x}\left(g\left(v_{2}, v_{3}\right)-g\left(v_{1}, v_{2}\right)\right) .
$$

The function $g$ defining the numerical flux is increasing with respect to the first variable and decreasing with respect to its second variable since the function $\beta$ is assumed to be decreasing. These two points prove that $H$ is increasing with the first and the last variable. It remains to show that it is also increasing with the second variable. For that purpose, we differentiate $H$ with respect to the second variable

$$
\partial_{v_{2}} H\left(\rho_{i-1}, v_{2}, \rho_{i+1}\right)=1-\frac{\Delta t}{\Delta x}\left(\beta\left(\rho_{i+1}\right)-\rho_{i-1} \beta^{\prime}\left(v_{2}\right)\right) .
$$

We observe that the CFL condition ensures the positivity of $\partial_{v_{2}} H$, and thus the monotonicity of our numerical scheme.

As a by-product of the consistency and monotonicity properties, we get the $L^{\infty}$-stability and the consistency with the entropy condition. We refer for these two points again to [13], Theorem 3.2 and Theorem 4.2 .

Stationary solutions. We are interested now in the determination of the stationary solutions of our model with the periodic boundary conditions. The stationary solutions are characterized by a constant flux, denoted $\phi$, such that

$$
\rho \beta(\rho)=\phi \quad \text { a.e.. }
$$

By definition of $\beta$, the flux function $\rho \in[0,1] \mapsto \rho \beta(\rho)$ is strictly increasing on $[0,1 / 2]$ from 0 to $1 / 4$ and strictly decreasing on $[1 / 2,1]$ from $1 / 4$ to 0 . Then, if $\phi<1 / 4$, there exist exactly two solutions to (9), say $\rho_{-}$and $\rho_{+}$, with $\rho_{-}<\frac{1}{2}<\rho_{+}$.

A unique "physical" (weak) solution to the conservation law is usually selected thanks to an additional entropy criterion (for the classical theory concerning classical entropy solutions we refer 
to Serre [19], and for applications to vehicular traffic, to Ansorge [2], Herbin, Leclercq [14]). If we assume moreover that our flux is strictly concave on $[0,1]$, which is the case for $\beta(\rho)=1-\rho$, then the Oleinik criterion selects the admissible stationary shocks

$$
\rho(t, x)=\left\{\begin{array}{ll}
\rho_{-} & \text {if } x<x^{*} \\
\rho_{+} & \text {if } x>x^{*}
\end{array}, \quad x^{*} \in(0,1) .\right.
$$

Therefore, with $\rho_{-}<\rho_{+}$, there are only three entropy stationary solutions of the conservation law which are

$$
\rho=\rho_{-}, \quad \rho=\rho_{+}, \quad \rho=\left\{\begin{array}{ll}
\rho_{-} & \text {if } x<x^{*} \\
\rho_{+} & \text {if } x>x^{*}
\end{array} .\right.
$$

This last case is possible only if $\rho_{-}\left(1-\rho_{-}\right)=\rho_{+}\left(1-\rho_{+}\right)=\phi \in(0,1 / 4)$.

As we have seen previously, our upwind/downwind numerical scheme satisfies a monotonicity property which ensures the consistency with the discrete entropy criterion. Therefore our numerical scheme should converge, as $\Delta x$ and $\Delta t$ go to 0 , towards the unique entropy solution of the system, and this is what is observed on the numerical simulations, as shown in Figure 5
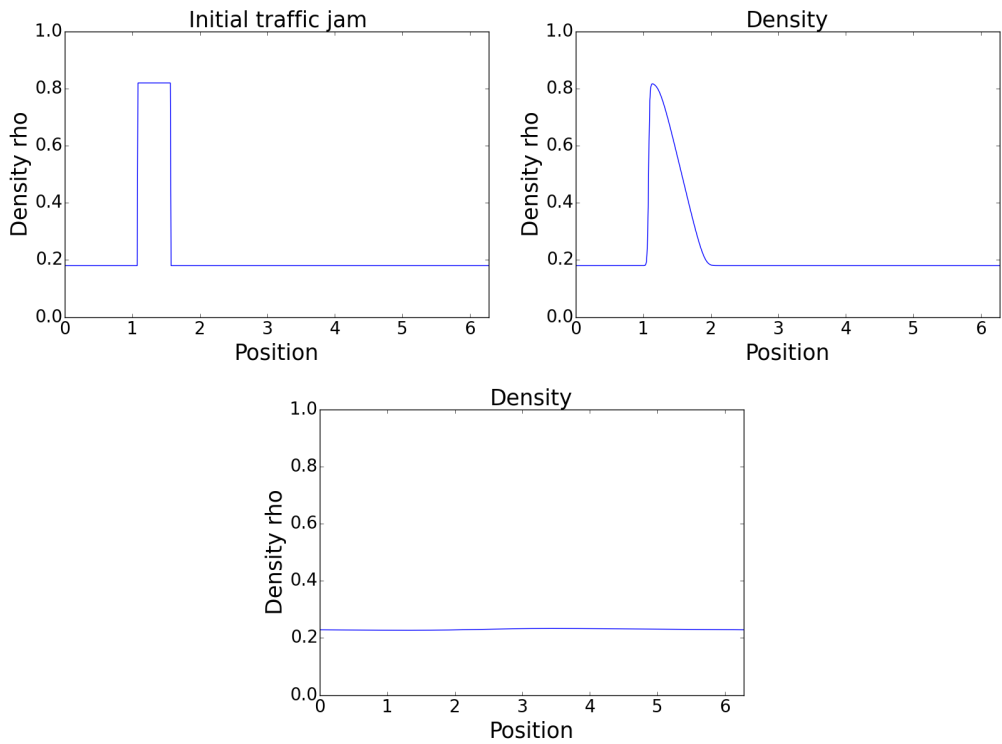

FiguRE 5. Initial traffic jam and relaxation towards a constant distribution, $v_{\max }=1$.

In order to investigate the properties of our scheme regarding stationnary solutions, we consider on the whole discrete space the semi-discrete equation: for all $i \in \mathbb{Z}$,

$$
\partial_{t} \rho_{i}+\frac{1}{\Delta x}\left(\rho_{i}\left(1-\rho_{i+1}\right)-\rho_{i-1}\left(1-\rho_{i}\right)\right)=0 .
$$

The stationary solutions of the previous equation are characterized by a constant flux $\phi \in[0,1 / 4]$ such that

$$
\rho_{i}\left(1-\rho_{i+1}\right)=\phi, \quad \forall i \in \mathbb{Z} .
$$

Proposition 2.1. Assume that $\phi \in(0,1 / 4)$ and consider the densities $\rho_{ \pm}=\frac{1 \pm \sqrt{1-4 \phi}}{2}$. If $\rho_{0}=1 / 2$, there exists a unique stationary solution of the scheme that corresponds to the flux $\phi$. It connects the state $\rho_{-}$at $-\infty$ to the state $\rho_{+}$at $+\infty$. There does not exist any stationary solution if we exchange $\rho_{-}$and $\rho_{+}$. 
Proof: If $\rho_{0}=1 / 2$, then

$$
\rho_{-i}=\frac{\phi}{1-\rho_{-i+1}}, \quad \forall i \in \mathbb{N}^{*},
$$

and the sequence converges as $i \rightarrow+\infty$ towards a fixed point of the map $g: r \mapsto \frac{\phi}{1-r}$, i.e. $\rho_{+}$ or $\rho_{-}$. Since

$$
g^{\prime}\left(\rho_{+}\right)=\frac{\phi}{\left(1-\rho_{+}\right)^{2}}=\frac{\rho_{+}}{1-\rho_{+}}>1, \quad \text { and } \quad\left|g^{\prime}\left(\rho_{-}\right)\right|=\frac{\rho_{-}}{1-\rho_{-}}<1
$$

we deduce that the sequence $\left(\rho_{-i}\right)_{i \geq 0}$ decreases towards $\rho_{-}$. In a symmetric way $\left(\rho_{i}\right)_{i \geq 0}$ converges towards $\rho_{+}$as $i \rightarrow+\infty$.

One can see that the sequence $\left(\rho_{i}\right)$ is nondecreasing, which excludes the non-entropic shocks.

We have exhibited in the previous section a microscopic stationary solution whose profile does not correspond to a stationary entropy solution of the conservation law (5). It suggests that the notion of entropy solutions may not be relevant in some particular situations observed in traffic flows. Some realistic situations, as our stable traffic jam, are indeed automatically rejected by the entropy criterion. Indeed, we need to take into account that a driver does not behave like a gas molecule and is not driven by a Brownian motion at the discrete level. In the rest of this section we propose a local modification of the upwind/downwind scheme (6) for the macroscopic conservation law in order to capture the potential stable traffic jams.

\subsection{Local modification of the numerical scheme}

We aim here at designing a local (one-cell) modification of the numerical scheme to capture the traffic jam observed at the microscopic level. To that purpose, we come back to the idea of the buffer zone introduced in the microscopic FTL model. It was a zone in which the cars have a constant velocity, and do not take into account the possible effects of congestion. Here we only modify how the information of congestion is taken into account on a given cell $i_{0}$ : instead of imposing a downwind discretization of the saturation function $\beta$, we discretize in an upwind manner the whole flux. By doing so we preserve the consistency of the scheme with the conservation law (5).

The scheme writes as

$$
\left\{\begin{array}{l}
\frac{\rho_{i}^{n+1}-\rho_{i}^{n}}{\Delta t}=\frac{1}{\Delta x}\left[\rho_{i-1}^{n}\left(1-\rho_{i}^{n}\right)-\rho_{i}^{n}\left(1-\rho_{i+1}^{n}\right)\right], \quad \forall i \neq i_{0}, i_{0}+1, \\
\frac{\rho_{i_{0}}^{n+1}-\rho_{i_{0}}^{n}}{\Delta t}=\frac{1}{\Delta x}\left[\rho_{i_{0}-1}^{n}\left(1-\rho_{i_{0}}^{n}\right)-\rho_{i_{0}}^{n}\left(1-\rho_{i_{0}}^{n}\right)\right], \\
\frac{\rho_{i_{0}+1}^{n+1}-\rho_{i_{0}+1}^{n}}{\Delta t}=\frac{1}{\Delta x}\left[\rho_{i_{0}}^{n}\left(1-\rho_{i_{0}}^{n}\right)-\rho_{i_{0}+1}^{n}\left(1-\rho_{i_{0}+2}^{n}\right)\right] .
\end{array}\right.
$$

Forgetting for a while the periodic boundary conditions, given a flux $\phi \in(0,1 / 4)$, one can check for instance that the discontinuity

$$
\rho_{i}= \begin{cases}\rho_{+} & \text {for } \quad i \leq i_{0} \\ \rho_{-} & \text {for } \quad i>i_{0}\end{cases}
$$

with $\rho_{-}$and $\rho_{+}$such that

$$
\rho_{-}\left(1-\rho_{-}\right)=\rho_{+}\left(1-\rho_{+}\right)=\phi
$$

is a stationary solution of the modified scheme. 
Let us look at the stability of this equilibrium. We denote the above stationary solution $\rho_{e}$ and we consider the perturbation

$$
\rho=\rho_{e}+\tilde{\rho} .
$$

The perturbation satisfies the equation

$$
\frac{\mathrm{d}}{\mathrm{d} t} \tilde{\rho}=\nabla F\left(\rho_{e}\right) \cdot \tilde{\rho}
$$

where $\nabla F\left(\rho_{e}\right)$ is a tridiagonal matrix

$$
\nabla F\left(\rho_{e}\right)=\frac{1}{\Delta x}\left(\begin{array}{ccccc}
b_{1} & c_{1} & 0 & \ldots & 0 \\
a_{2} & b_{2} & c_{2} & \ddots & \vdots \\
0 & \ddots & \ddots & \ddots & 0 \\
\vdots & \ddots & a_{N-1} & b_{N-1} & c_{N-1} \\
0 & \ldots & 0 & a_{N} & b_{N}
\end{array}\right) .
$$

with

$$
\begin{gathered}
a_{i}=1-\rho_{i}, \quad b_{i}=-1, \quad c_{i}=\rho_{i}, \quad \forall i \neq i_{0}, i_{0}+1, \\
a_{i_{0}}=1-\rho_{+}, \quad b_{i_{0}}=-1+\rho_{+}, \quad, \quad c_{i_{0}}=0, \\
a_{i_{0}+1}=1-2 \rho_{+}, \quad b_{i_{0}+1}=-1+\rho_{-}, \quad c_{i_{0}+1}=\rho_{-} .
\end{gathered}
$$

Thanks to the Gershgorin circle theorem, we deduce that the eigenvalues are located in one of the following disks

$$
\mathrm{D}(-1,1), \quad \mathrm{D}\left(-1+\rho_{+}, 1-\rho_{+}\right), \quad \mathrm{D}\left(-1+\rho_{-}, 1-2 \rho_{+}+\rho-\right) .
$$

Since, $\rho_{-}<\rho_{+}$, none of these disks intersects the half plane of positive real part, except in 0 , which gives the stability in $L^{2}$ of $\rho_{e}$ (the eigenvalue 0 corresponds to a global translation).

We argue that this numerical modification is the macroscopic counterpart of the buffer zone, or, in other words, that it is the residual microscopic effect of the buffer zone at the continuous limit. We can numerically compare the microscopic simulation with the buffer zone and the macroscopic simulation with local modification on one cell whose position corresponds to the discrete position of the buffer zone exit. To do so we start with a macroscopic initial density which is discretized theoretically via the operator (c.f. [8])

$$
E_{N}: \rho \mapsto\left\{\begin{array}{l}
x_{N}=2 \pi \\
x_{i}=\max \left\{x<x_{i+1}, \int_{x}^{x_{i+1}} \rho(y) \mathrm{d} y=\delta_{N}^{*}\right\}, \quad \forall i \in\{1, \ldots, N-1\}
\end{array} .\right.
$$

where $\delta_{N}^{*} N=M$, if the initial density $\rho^{0}$ satisfies $\int \rho^{0}(x) \mathrm{d} x=M$. Thanks to this local modification of the numerical flux, we are able to capture the stable traffic jam, equivalent to the one obtained with the microscopic coupled FTL-buffer zone scheme. The numerical results are shown on Figure 6 .

Stability under perturbations. In order to test the stability of a traffic jam we consider two types of perturbations: first a "physical perturbation" based on microscopic considerations, and then a random one.

The physical perturbations that we impose on the downward jam consists in perturbing two neighbour cells by the same amplitude, but with opposite signs. Doing so, we ensure that the perturbed density has the same total mass as before. The density on the buffer cell remains unchanged. Physically, this perturbation expresses the following fact: if a driver reduces his distance to his predecessor, he will also increase the distance to his successor. The numerical results are represented on Figure 7. Both on microscopic and macroscopic simulations we recover after some time the traffic jam. The jam appears to be stable under such perturbations which keep unchanged the density at the buffer cells. 

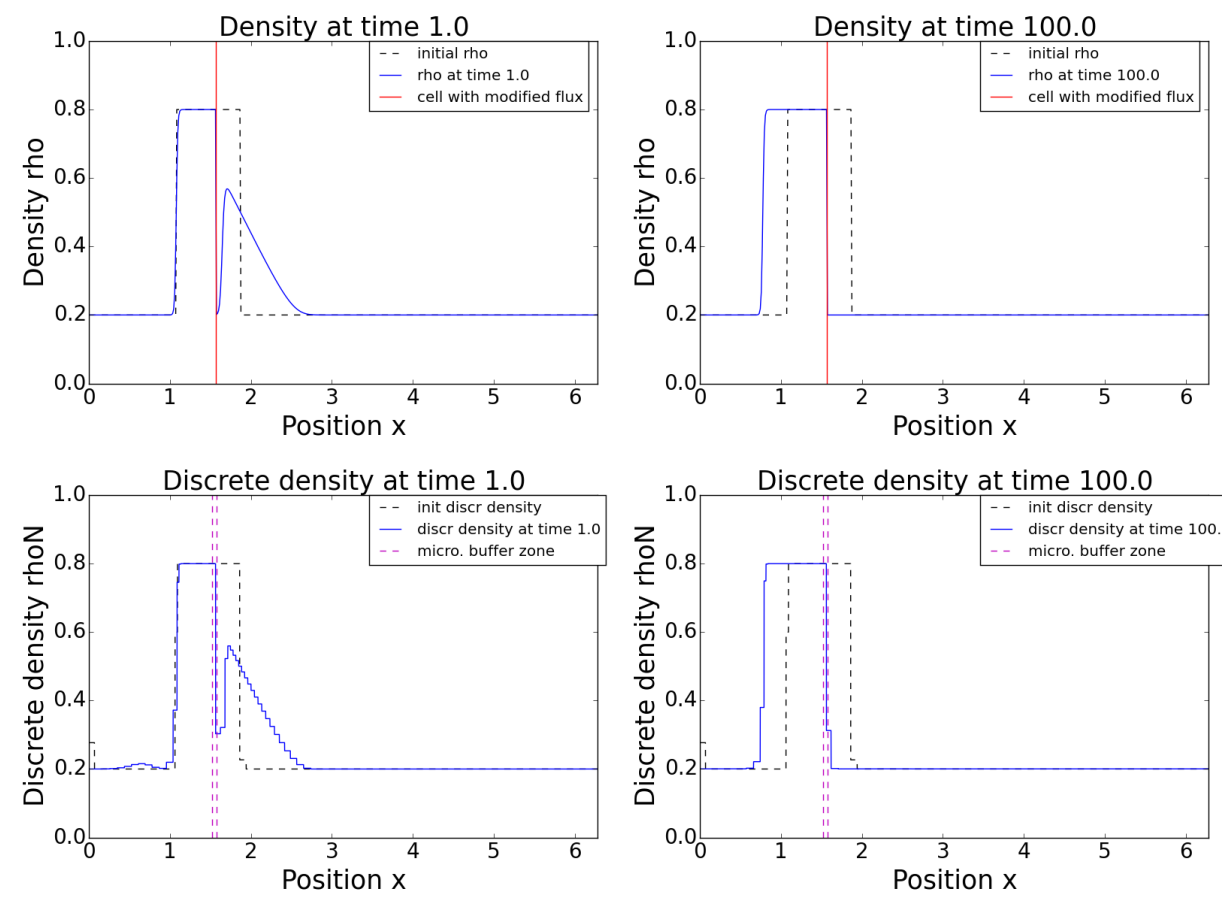

FiguRE 6. Stable jam from macroscopic (top) and microscopic (bottom) point of view. Parameters: $N=96, v_{\max }=1$ and $\delta^{*}=0.018$.

Remark: The asymptotic behaviour of the solution is very sensitive to the initial density. As represented on Figure 8 , if for instance we modify a little bit the value of the density on the buffer cell, the jam may disappear and the solution converges towards a constant state.

Now, if we perturb the initial data randomly, stable traffic jams appear and are persistent. On Figure 9 we compare three random initial data with same mass but for different values in the neighbourhood of the buffer cell. We observe that different stable traffic jams are obtained at time $T=200$. The jam is then characterized by the jump at the buffer cell. It seems then that even for small random perturbations of a stable traffic jam, we can obtain a different stable solution if the jump just after the buffer cell is modified.

Thus, for a fixed total mass, the numerical simulations indicate that there exists a continuum of possible macroscopic traffic jams, characterized by the jump at the buffer cell. They seem stable under perturbations which do not affect the density in the neighbourhood of the buffer cell.

\section{REFERENCES}

[1] B. Andreianov, P. Goatin, and Nicolas Seguin. Finite volume schemes for locally constrained conservation laws. Numerische Mathematik, 115.4, pp. 609-645,(2010).

[2] R. Ansorge. What does the entropy condition mean in traffic flow theory? Transportation Research Part B: Methodological, 24(2), pp. 133-143, (1990).

[3] A. Aw, Klar, M. Rascle, and T. Materne. Derivation of continuum traffic flow models from microscopic follow-the-leader models. SIAM Journal on Applied Mathematics, 63 (1), pp. 259-278, (2002).

[4] N. Bellomo and C. Dogbe. On the modelling of Traffic and Crowds: A Survey of Models, Speculations, and Perspectives. SIAM Review, 53 (3), pp. 409-463, (2011).

[5] R. E. Chandler, R. Herman and E. W. Montroll. Traffic Dynamics: Studies in Car Following. Operations Res., 6, pp. 165-184, (1958).

[6] R. Colombo and P. Goatin. A well posed conservation law with a variable unilateral constraint. Journal of Differential Equations 234.2, pp. 654-675, (2007).

[7] R. Colombo, and M. Rosini. Pedestrian flows and non-classical shocks. Mathematical Methods in the Applied Sciences 28.13, pp. 1553-1567, (2005). 

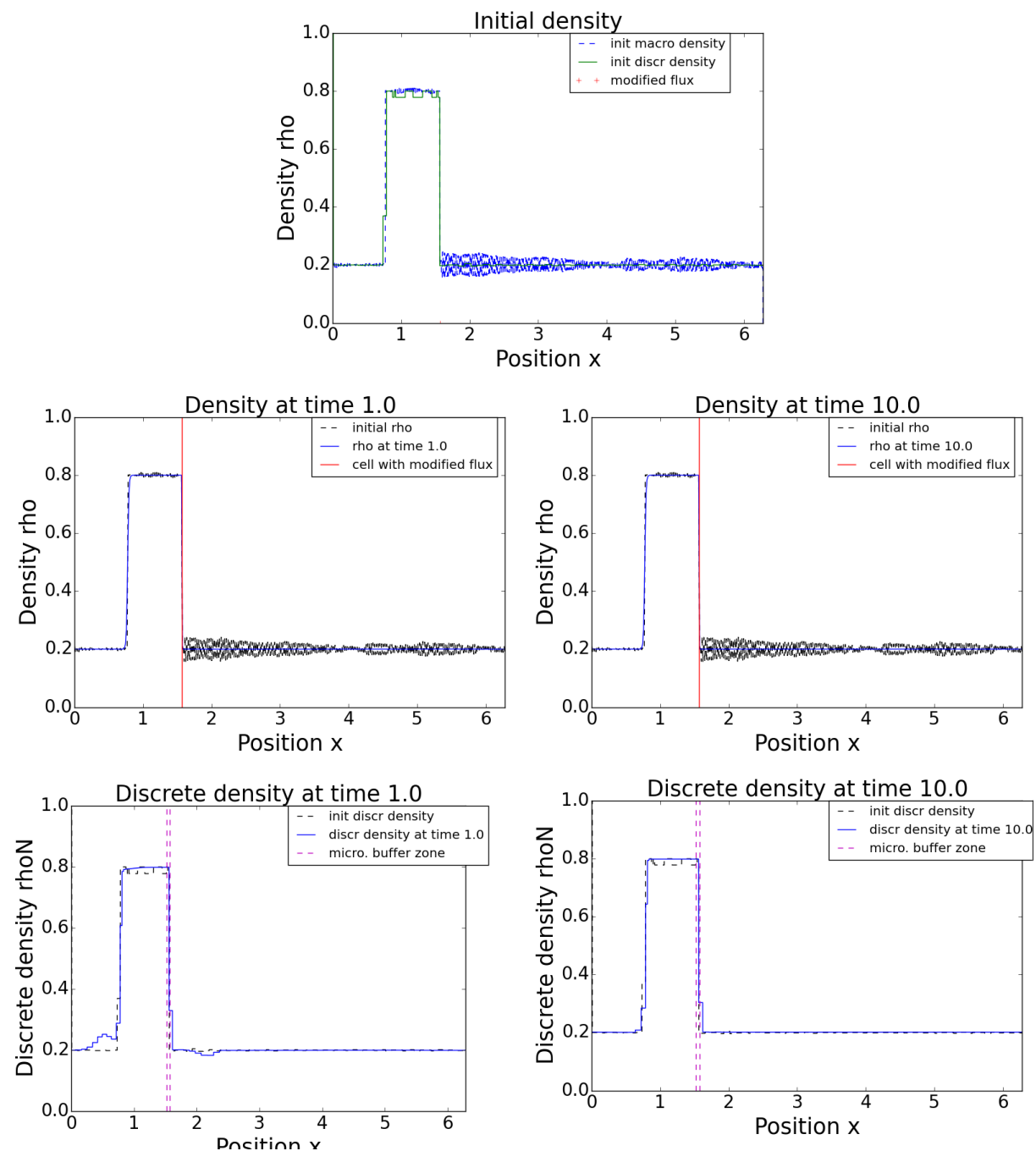

Figure 7. Perturbed initial density and convergence towards the stable jam from macroscopic and microscopic point of view. Parameters: $N=96, v_{\max }=1$ and $\delta^{*}=0.018$.

[8] R. M. Colombo and E. Rossi. On the Micro-Macro Limit in Traffic Flow. Rend. Semin. Mat. Univ. Padova, 131, pp. 217-235, (2014).

[9] N. Forcadel, C. Imbert, and R. Monneau. Homogenization of some particle systems with two-body interactions and of the dislocation dynamics. Discrete and Continuous Dynamical Systems-Series A, 23, pp. 785-826, (2009).

[10] N. Forcadel, and W. Salazar. A junction condition by specified homogenization of a discrete model with a local perturbation and application to traffic flow. to appear, (2014).

[11] M. Di Francesco and M. D. Rosini. Rigorous derivation of nonlinear scalar conservation laws from followthe-leader type models via many particle limit. Arch. Rational Mech. Anal., 217, pp. 831-871, (2015).

[12] D. C. Gazis, R. Herman and R. Rothery. Nonlinear follow-the-leader models of traffic flow. Operations Res., 9, pp. 545-567, (1961).

[13] E. Godlewski, P. A. Raviart. Hyperbolic systems of conservation laws. Springer, Mathématiques et applications, Ellipses, Paris, (1991).

[14] R. Herbin, L. Leclercq. "A note on the Entropy Solutions of the Hydrodynamic Model of Traffic Flow" revisited. Transportation Science, 45(1), pp. 138-142, (2011).

[15] A. Jelic̀, C. Appert-Rolland, S. Lemercier, J. Pettré. Properties of pedestrians walking in line Fundamental diagrams. Phys. Rev. E, 85 036111, (2012)

[16] M. J. Lighthill, G. B. Whitham. On kinematic waves II: A theory of traffic flow on long, crowded roads. Proceedings of The Royal Society of London Ser. A , 229, pp. 317-345, (1955). 

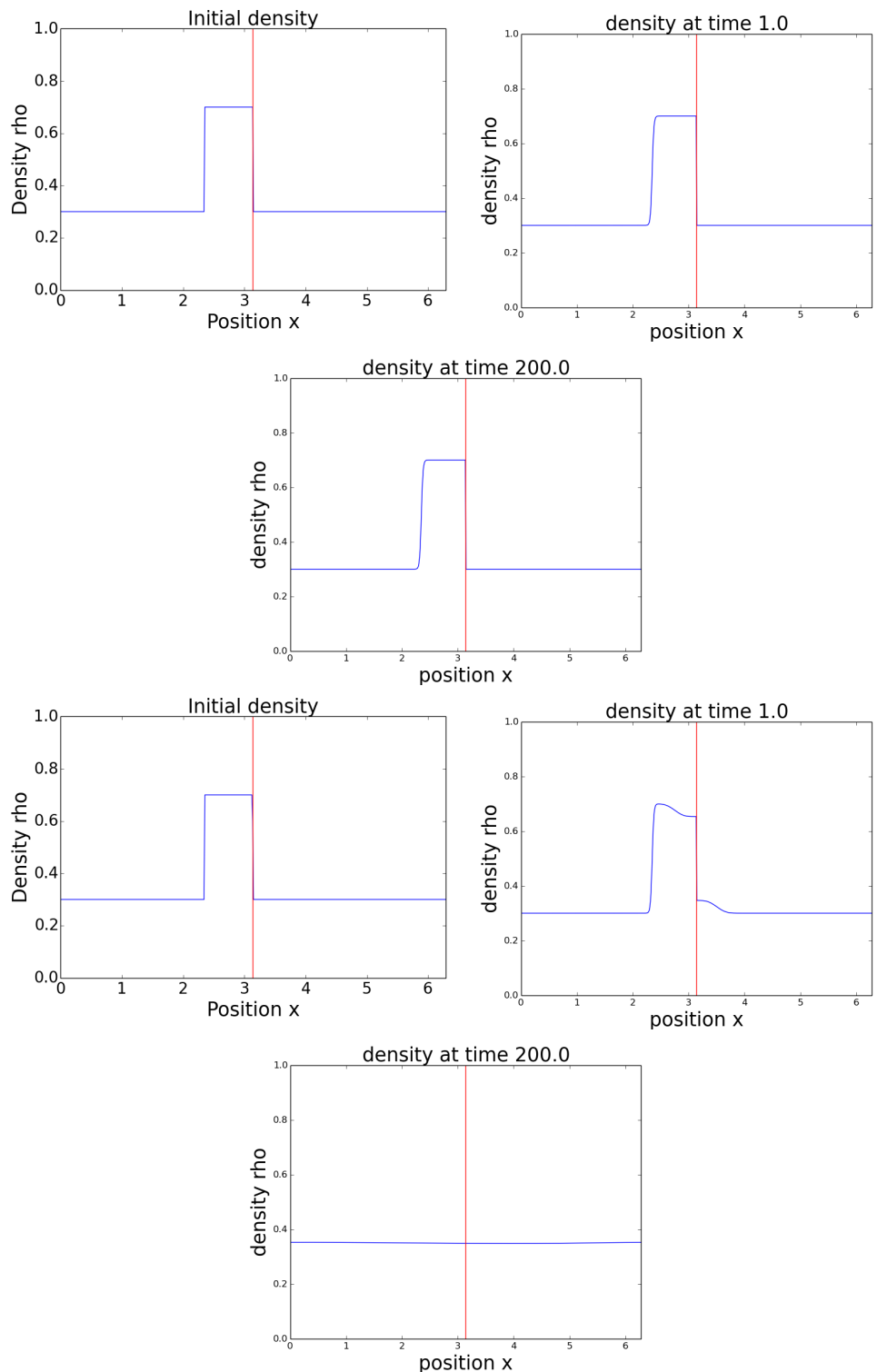

Figure 8. Comparison between two initial data differing only by the value on the buffer cell and leading to different stationary states. Top: $\rho^{0}\left[i_{0}\right]=\rho_{+}=0.7$, bottom: $\rho^{0}\left[i_{0}\right]=0.6$.

[17] N. Polson, V. Sokolov. Bayesian analysis of traffic flow on interstate I-55: The LWR model. The Annals of Applied Statistics 9, no. 4, pp. 1864-1888, (2015).

[18] P. I. Richards. Shock waves on the highway. Operations Research, 4, pp. 42-51, (1956).

[19] D. Serre. Systems of conservation laws. 1 \& 2. Cambridge University Press, Cambridge, (1999). 

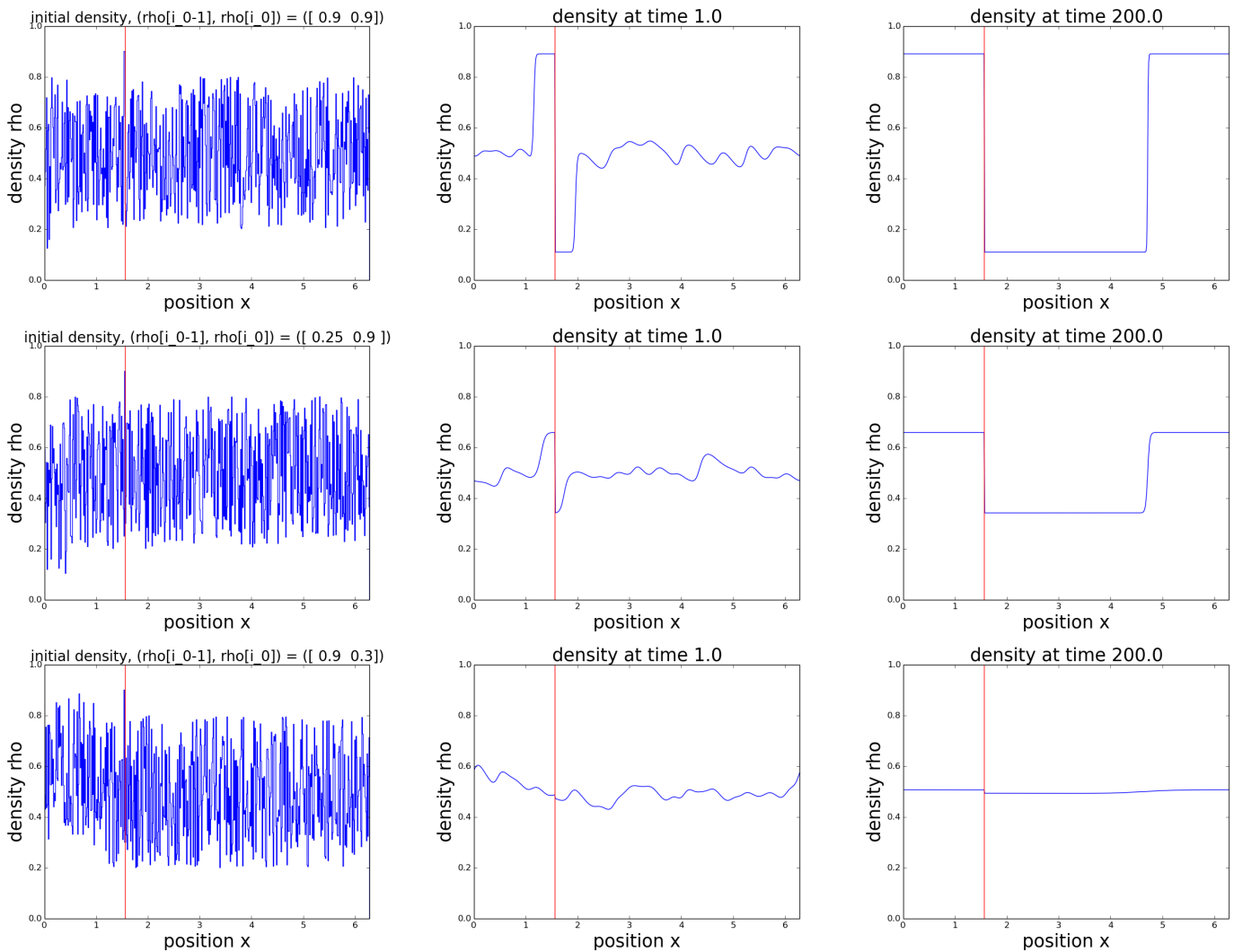

FIgURE 9. Comparison between random initial data of same mass 0.5 